\title{
Resummations in the Bloch-Nordsieck model
}

\author{
A. Jakovád and P. Mati \\ Institute of Physics, Budapest University of Technology and Economics, H-1111 Budapest, Hungary
}

\begin{abstract}
We studied different levels of resummations of the exactly solvable Bloch-Nordsieck model in order to be able to compare the approximations with an exact result. We studied one-loop perturbation theory, 2PI resummation and Schwinger-Dyson equations truncated in a way to maintain Wardidentities. At all levels we carefully performed renormalization. We found that although the 2PI resummation does not exhibit infrared (IR) sensitivity at the mass shell, as the one-loop perturbation theory does, but it is still far from the exact solution. The method of truncated Schwinger-Dyson equations, however, is exact in this model, so it provides a new way of solving the Bloch-Nordsieck model. This method can also be generalized to other, more complicated theories.
\end{abstract}

\section{INTRODUCTION}

In field theories we often encounter infrared (IR) divergences of different kind. Some of them have physical meaning while others are just artifacts of the perturbation theory. A common feature, however, is that the IR divergences appear at each order of perturbation theory, usually being more and more severe at higher loop orders. In order to consistently define the theory, these IR divergences must be summed up.

2PI resummations provide a consistent resummation framework known for a long time [1]. The basic idea is to replace the free propagator in the perturbation theory with the exact one which is approximated self-consistently with fixed-loop skeleton diagrams. The so-defined perturbation theory is renormalizable [2]-[12], and can be applied to study different physical questions from non-equilibrium [8], [9], thermodynamics [6], [10], 14], [15] and different systems like $\mathrm{O}(\mathrm{N})$ model [11], 12] or gauge theories [13].

Although the 2PI approximation is constructed by physical arguments, and we expect better results (ie. closer to the exact one) after 2PI resummation, a priory it is not sure that one really achieves this goal. Probably the finite lifetime effects are well represented by 2PI resummation both in equilibrium [6] as well in non-equilibrium, where the 2PI is close to the Boltzmann-equation approximation [16]. But if the deep IR regime is important where multi-particle excitations also play crucial role, the picture is far to be so clean. To make the case even worse, in most gauge theory models there is hard to make exact statements about the IR behavior of the model.

In this paper we aim to study the Bloch-Nordsieck model, which is an exactly solvable $3+1 \mathrm{D}$ gauge theory [17]. It is the eikonal approximation model of the QED, and one can argue [19] that in the deep IR regime it describes correctly QED. Therefore it is used to estimate IR properties of QED from this model, for example the soft photon creation processes [20] or finite temperature electron damping [19].

This model is therefore a motivated case study where the accuracy of the 2PI resummation can be assessed. We therefore perform a series of approximations for the Bloch-Nordsieck model: a one-loop perturbation theory, a 2PI resummation and finally the solution of the Schwinger-Dyson equations with an Ansatz for the vertex function. In this model all levels of the approximations can be treated analytically. We show that the last method is exact in the model under consideration - although that is not expected in general. This observation, however, leads us to a proposal how the $2 \mathrm{PI}$ resummation can be improved in a generic model in order to catch the IR physics correctly.

The structure of the paper is as follows. We first overview the standard solution of the Bloch-Nordsieck propagator at zero temperature in Section [II Then we compute the one loop level fermion propagator in Section [II] Next, in Section IV we write up the 2PI equations for this model, perform renormalization on that, and give the analytical solution as far it can be done. Then we study the problem also numerically, determine the fermion propagator and compare the result with the exact one. Finally, in Section $\nabla$ we study the truncated Schwinger-Dyson equations, renormalize it, and show that for the Bloch-Nordsieck model the so-defined approximation scheme is exact. For conclusion (Section VI) we summarize our approach again and speculate about the possible generalizations.

\footnotetext{
* jakovac@phy.bme.hu

† mati@phy.bme.hu
} 


\section{THE MODEL AND THE EXACT SOLUTION}

The Bloch-Nordsieck model is a simplification of the QED Lagrangian where the Dirac matrices $\gamma^{\mu}$ are replaced by a four-vector $u^{\mu}$

$$
\mathcal{L}=-\frac{1}{4} F_{\mu \nu} F^{\mu \nu}+\bar{\Psi}\left(i u_{\mu} D^{\mu}-m\right) \Psi, \quad i D_{\mu}=i \partial_{\mu}-e A_{\mu}, \quad F_{\mu \nu}=\partial_{\mu} A_{\nu}-\partial_{\nu} A_{\mu}
$$

The singled-out four vector $u_{\mu}$ represents the velocity of the rest frame of the fermion. The fermion wave function here has only one component and $\bar{\Psi}=\Psi^{*}$.

We are interested in the fermion propagator which reads in the path integral representation

$$
i \mathcal{G}(x)=\langle T \Psi(x) \bar{\Psi}(0)\rangle=\frac{1}{Z} \int \mathcal{D} \bar{\Psi} \mathcal{D} \Psi \mathcal{D} A_{\mu} e^{i S[\bar{\Psi}, \Psi, A]} \Psi(x) \bar{\Psi}(0) .
$$

At the tree level it reads:

$$
\mathcal{G}_{0}(p)=\frac{1}{u_{\mu} p^{\mu}-m+i \varepsilon}
$$

Since it has a single pole, there is no antiparticles in the model, and also the Feynman propagator is the same as the retarded propagator. The lack of antiparticles also means that all closed fermion loops are zerd 1 . As a consequence the photon self-energy is zero, the free photon propagator is the exact one. In Feynman gauge therefore the exact photon propagator is

$$
G_{\mu \nu}(k)=\frac{-g_{\mu \nu}}{k^{2}+i \varepsilon}
$$

Now we shortly review the exact solution for the fermion propagator, cf. [17], [18]. We first define the gauge field dependent propagator:

$$
\mathcal{G}(x ; A)=\int \mathcal{D} \bar{\Psi} \mathcal{D} \Psi e^{i S[\bar{\Psi}, \Psi, A]} \Psi(x) \bar{\Psi}(0) .
$$

This satisfies the Schwinger-Dyson equation

$$
\left(i u_{\mu} \partial^{\mu}-e u_{\mu} A^{\mu}-m\right) \mathcal{G}(x ; A)=-\delta(x) .
$$

We will need the solution in an exponential form for $A$, and this is achieved by introducing the auxiliary quantity $U(x, \nu)$ which satisfies

$$
i \frac{\partial U(x, \nu)}{\partial \nu}=\left(i u_{\mu} \partial^{\mu}-e u_{\mu} A^{\mu}-m\right) U(x, \nu), \quad U(x, 0)=\delta(x) .
$$

By integration of the above equation by $\nu$ and assuming $U(x, \infty)=0$ (for which we need a convergence factor $i \varepsilon$ ) we see that

$$
\mathcal{G}(x)=-i \int d \nu U(x, \nu)
$$

We perform Fourier transformation and separate the free time dependence $U(p, \nu)=e^{-i\left(u_{\mu} p^{\mu}-m\right) \nu} \bar{U}(p, \nu)$, then we obtain

$$
\frac{\partial \bar{U}(p, \nu)}{\partial \nu}=i e u_{\mu} \int \frac{d^{4} k}{(2 \pi)^{4}} A^{\mu}(p-k) e^{i u^{\mu}\left(p_{\mu}-k_{\mu}\right)} \bar{U}(k, \nu), \quad \bar{U}(p, 0)=1 .
$$

The linear $p_{\mu}-k_{\mu}$ behavior is the consequence of the linearity in the kernel. If the kernel is non-linear or it is not scalar (has a matrix structure) then this form is not true any more. From here an inverse Fourier transformation yields

$$
\frac{\partial \bar{U}(x, \nu)}{\partial \nu}=i e u_{\mu} A^{\mu}(x+u \nu) \bar{U}(x, \nu) \quad \Rightarrow \quad \bar{U}(x, \nu)=e^{\int_{0}^{\nu} d \nu^{\prime} A\left(u \nu^{\prime}\right)} \delta(x)
$$

\footnotetext{
1 This statement can be best seen in real time representation. There a chain of fermion propagators, because of the retardation, is proportional to $\Theta\left(t_{1}-t_{2}\right) \ldots \Theta\left(t_{n-1}-t_{n}\right)$. In a closed loop $t_{n}=t_{1}$, therefore the product of theta functions is zero almost everywhere.
} 
Once we have an exponential representation for the background-dependent propagator, we can perform the Gaussian $A$-integration. As a result we obtain in the exponent the factor

$$
\frac{i e^{2}}{2} \int \frac{d^{4} k}{(2 \pi)^{4}} R_{\nu}^{*}(k) G_{\mu \nu}(k) R_{\nu}(k), \quad R_{\nu}(k)=\int_{0}^{\nu} d \nu^{\prime} e^{-i k_{\mu} u^{\mu} \nu^{\prime}} .
$$

This integral is UV divergent; in dimensional regularization one finds the result

$$
\frac{\alpha}{2 \pi \varepsilon}+\frac{\alpha}{\pi} \ln i \bar{\mu} \nu
$$

where $\alpha=e^{2} /(4 \pi)$ and $\bar{\mu}=\sqrt{4 \pi} e^{\gamma_{E}} \mu$. Then the fermion propagator reads

$$
\mathcal{G}(p)=-i e^{\frac{\alpha}{2 \pi \varepsilon}} \int_{0}^{\infty} d \nu e^{-i \nu\left(u_{\mu} p^{\nu}-m\right)+\frac{\alpha}{\pi} \ln i \bar{\mu} \nu}=\frac{Z}{\left(u_{\mu} p^{\nu}-m\right)^{1+\frac{\alpha}{\pi}}}
$$

where $Z=\Gamma\left(1+\frac{\alpha}{\pi}\right) e^{\frac{\alpha}{2 \pi \varepsilon}} \bar{\mu}^{\frac{\alpha}{\pi}}$. This is UV divergent which means that we need a wave function renormalization. The renormalized propagator reads

$$
\mathcal{G}_{r e n}(p)=\frac{\zeta}{\left(u_{\mu} p^{\nu}-m\right)^{1+\frac{\alpha}{\pi}}}
$$

where $\zeta$ is a finite quantity.

We can determine the discontinuity of this formula, for simplicity choosing $u_{\mu}=(1,0,0,0)$ :

$$
\varrho(p)=\operatorname{Disc}_{p_{0}} \mathcal{G}(p)=\Theta\left(p_{0}-m\right) \frac{\zeta\left(1-e^{2 i \alpha}\right)}{\left(p_{0}-m\right)^{1+\frac{\alpha}{\pi}}} .
$$

With this spectral function the sum rule $\int_{-\infty}^{\infty} d p_{0} \varrho\left(p_{0}\right)=1$, which is the consequence of the equal time anticommutation relations, cannot be fulfilled, since the integral is divergent. This divergence should be compensated with the choice $\zeta=0$, but then we are faced with a $0 \times \infty$ expression. Therefore one should always use a regularized version of the spectral function (or propagator), maintaining the sum rule, and only at the end of the calculation is one allowed to release the regularization.

The Lagrangian is Lorentz-invariant in the sense that we must also transform $u$. So we can choose a Lorentztransformation where $\Lambda u=\left(u_{0}, 0,0,0\right)$. If $u^{\mu}$ is a 4 -velocity then $u_{0}=1$; if it is of the form $u=(1, \mathbf{v})$, then it is $u_{0}=\sqrt{1-\mathbf{v}^{2}}$. After rescaling the field $\Psi \rightarrow \Psi / \sqrt{u_{0}}$ and the mass as $m \rightarrow u_{0} m$, the Lagrangian reads

$$
\mathcal{L}=-\frac{1}{4} F_{\mu \nu} F^{\mu \nu}+\bar{\Psi}\left(i D_{0}-m\right) \Psi
$$

This Lagrangian will be used mostly in this work later. If necessary, the complete $u$ dependence is easily recoverable.

\section{ONE LOOP PERTURBATION THEORY}

The goal of our investigations is to see, how the different levels of resummations improve the result. Thus first we start with the one loop perturbation theory. Here we need the renormalized Lagrangian; in Feynman gauge it reads (using the fact that the photon self-energy is zero):

$$
\mathcal{L}=-\frac{1}{2}\left(\partial_{\mu} A_{\nu}\right)^{2}+\bar{\Psi}\left(i \partial_{0}-m\right) \Psi-e \bar{\Psi} A_{0} \Psi+\delta Z \bar{\Psi} i \partial_{0} \Psi-\delta Z_{m} m \bar{\Psi} \Psi-\delta e \bar{\Psi} A_{0} \Psi
$$

For the fermion self-energy the one loop diagram is the bubble with the contribution:

$$
-i \Sigma_{1 \text { loop }}(p, m)=(-i e)^{2} \int \frac{d^{4} k}{(2 \pi)^{4}} i G_{00}(k) i \mathcal{G}(p-k)=-e^{2} u^{2} \int \frac{d^{4} k}{(2 \pi)^{4}} \frac{1}{k^{2}+i \varepsilon} \frac{1}{p_{0}-k_{0}-m+i \varepsilon} .
$$

Moreover we have wave function and mass renormalization counterterms

$$
\Sigma_{c t}(p)=-\delta Z p_{0}+\delta Z_{m} m
$$


The complete one loop self-energy is $\Sigma_{1 \text { loop }}+\Sigma_{c t}$. In the calculation we have to take care of the non-standard form of the free fermion propagator. The details of the computation can be found in the Appendix, as a result we obtain

$$
\Sigma_{1 \text { loop }}(p, m)=\frac{\alpha}{\pi}\left(p_{0}-m\right)\left[-\ln \frac{m-p_{0}}{\mu}+\mathcal{D}_{\varepsilon}\right],
$$

where $\alpha=e^{2} /(4 \pi)$ and

$$
\mathcal{D}_{\varepsilon}=\frac{1}{2 \varepsilon}+1+\frac{1}{2}\left(\ln \pi-\gamma_{E}\right)
$$

For renormalization we have to subtract the divergences with help of the counterterms, the finite parts are fixed by the renormalization scheme. In the $\overline{\mathrm{MS}}$ scheme we choose the counterterms like

$$
\delta Z_{1, \overline{\mathrm{MS}}}=\delta Z_{m, \overline{\mathrm{MS}}}=\frac{\alpha}{\pi} \mathcal{D}_{\varepsilon}
$$

this results in

$$
\Sigma_{r e n}(p)=-\frac{\alpha}{\pi}\left(p_{0}-m\right) \ln \frac{m-p_{0}}{\mu} .
$$

The discontinuity of the renormalized self-energy reads

$$
\underset{p_{0}}{\operatorname{Disc}} \Sigma(p)=2 \alpha\left(p_{0}-m\right) \Theta\left(p_{0}-m\right)
$$

For the one-loop propagator we obtain

$$
\mathcal{G}(p)=\frac{1}{p_{0}-m-\Sigma(p)}=\frac{1}{p_{0}-m} \frac{1}{1+\frac{\alpha}{\pi} \ln \frac{m-p_{0}}{\mu}} .
$$

This is consistent with the exact result (14) in the leading order of $e^{2}$.

The spectral function $\operatorname{Disc}_{p_{0}} i \mathcal{G}$ reads

$$
\varrho(p)=\frac{\Theta\left(p_{0}-m\right)}{p_{0}-m} \frac{2 \alpha}{\left(1+\frac{\alpha}{\pi} \ln \frac{p_{0}-m}{\mu}\right)^{2}+\alpha^{2}} .
$$

This spectral function is normalizable, since

$$
\int_{-\infty}^{\infty} \frac{d p_{0}}{2 \pi} \varrho(p)=\frac{\pi}{\alpha}
$$

On the other hand the one-loop result is not reliable when $\left|\ln \left(p_{0}-m\right) / \mu\right| \gg \frac{\pi}{\alpha}$, ie. in the vicinity of the mass shell as well as in the large $p_{0}$ regime. In order to have a better description of these kinematical regimes, we need resummation of certain class of diagrams.

\section{2PI RESUMMATION}

As it is discussed in the Introduction, the next level of our approximations is the 2PI resummation. The idea is to use the exact propagators in the perturbation theory, this propagator is determined self-consistently using skeleton diagrams as resummation patterns. The one-loop bubble diagram in the present case generates the resummation of all the "rainbow" diagrams. To obtain an expression for the 2PI resummation we use the technique of [6] : we use the 1loop formula (18), interpret the appearing propagators as full propagators, and finally perform renormalization with the same form of divergent parts of the counterterms as in the 1-loop case (the actual values will be different).

The tree level photon propagator is exact, therefore we can write

$$
\Sigma(p)=-i e^{2} \int \frac{d^{4} k}{(2 \pi)^{4}} \frac{\mathcal{G}(p-k)}{k^{2}+i \varepsilon} .
$$


Using a spectral representation for the fermion propagator (using that now the Feynman propagator is the retarded one and that the fermion spectral function is $\varrho(\omega<0)=0)$ we find

$$
\Sigma(p)=-i e^{2} \int_{0}^{\infty} \frac{d \omega}{2 \pi} \int \frac{d^{4} k}{(2 \pi)^{4}} \frac{1}{k^{2}+i \varepsilon} \frac{\varrho(\omega)}{p_{0}-k_{0}-\omega+i \varepsilon} .
$$

From this form it is clear that we obtain the weighted one-loop result, ie.

$$
\Sigma(p)=\int_{0}^{\infty} \frac{d \omega}{2 \pi} \varrho(\omega) \Sigma_{1 l o o p}(p, \omega)
$$

In particular, if $\varrho(\omega)=2 \pi \delta(\omega-m)$, then we get back the one-loop result.

At this point it is worth to examine the UV divergence structure of the 2PI approximation. UV divergences may occur in (30) for large values of $\omega$ : using (20) we find that the large $\omega$ behavior of the one-loop self-energy reads:

$$
\Sigma_{1 \text { loop }}(p, \omega)=\frac{\alpha}{\pi} \omega\left(\ln \frac{\omega}{\mu}-\mathcal{D}_{\varepsilon}\right)+\frac{\alpha}{\pi}\left(-\ln \frac{\omega}{\mu}+\mathcal{D}_{\varepsilon}\right) p_{0}+\mathcal{O}\left(\frac{p_{0}^{2}}{\omega}\right) .
$$

Since $\varrho$ is integrable for large $\omega$ values, therefore the $\mathcal{O}\left(\omega^{-1}\right)$ is already finite. Therefore the divergence structure of the self-energy is $A+B p_{0}$, just like for the free case, and so the same type of counterterms are needed (although the values are different). This is a manifestation of the general case of counterterm renormalizability of $2 \mathrm{PI}$ resummations [6].

\section{A. Analytic study of the 2PI equations}

First we try to analyze (30) with analytic methods. We differentiate it with respect to $p_{0}$ to find

$$
\frac{\partial \Sigma_{1 \text { loop }}}{\partial p_{0}}=\frac{\alpha}{\pi}\left(-\ln \frac{\omega-p_{0}-i \varepsilon}{\mu}-1\right), \quad \frac{\partial^{2} \Sigma_{1 l o o p}}{\partial p_{0}^{2}}=-\frac{\alpha}{\pi} \frac{1}{p_{0}-\omega+i \varepsilon}, \quad \frac{\partial^{2} \Sigma}{\partial p_{0}^{2}}=-\frac{\alpha}{\pi} \mathcal{G} .
$$

Since $\mathcal{G}^{-1}=p_{0}-m-\Sigma$, we find for $\mathcal{G}^{-1}$ :

$$
\frac{d^{2} \mathcal{G}^{-1}}{d p_{0}^{2}} \mathcal{G}^{-1}=\frac{\alpha}{\pi}
$$

To solve the equation we first should realize that the $\alpha=0$ and $\alpha \neq 0$ cases are very different. If $\alpha=0$ then $\left(\mathcal{G}^{-1}\right)^{\prime \prime}=0$ and the propagator behaves as $\mathcal{G}=Z /\left(p_{0}-\tilde{m}\right)$ with some wave function renormalization constant $Z$ and mass $\tilde{m}$. This agrees with the free case. We also see that the integration constants correspond to the renormalization scheme (here the wave function and mass renormalization).

If $\alpha \neq 0$ then we can redefine the variables with an arbitrary $\mathcal{G}_{0}$ scale as

$$
E=\mathcal{G}_{0} \sqrt{\frac{2 \alpha}{\pi}}\left(m-p_{0}\right), \quad \Psi=-\mathcal{G}_{0} \mathcal{G}^{-1},
$$

then we find

$$
2 \frac{d^{2} \Psi}{d E^{2}} \Psi=1
$$

This equation does not depend on the coupling any more. The coupling constant dependence shows up in the integration constants which are the manifestation of the renormalization scheme. We shall also note that the equation does not give information about the sign of $E$ and $\Psi$, because for $E \rightarrow-E$ or $\Psi \rightarrow-\Psi$ the equation remains the same. The chosen signs in (34) turn out later to be the physical choice.

We introduce

$$
y=\frac{d \Psi}{d E} \Rightarrow \frac{d y}{d E}=\frac{d y}{d \Psi} \frac{d \Psi}{d E}=E \frac{d y}{d \Psi} .
$$


This means that we can write for $y$ :

$$
2 y \Psi \frac{d y}{d \Psi}=1 \quad \Rightarrow \quad y=\frac{d \Psi}{d E}=\sqrt{\ln \Psi}+y_{0},
$$

with an integration constant $y_{0}$. Therefore

$$
\int_{1}^{\Psi} \frac{d \Psi^{\prime}}{\sqrt{\ln \Psi^{\prime}}+y_{0}}=E
$$

There could appear an integration constant also here on the right hand side: $E-E_{0}$. But recalling that $E \sim p_{0}-m$, we see that $E_{0}$ corresponds to a mass shift: if the mass remains the tree level $m$ then $E_{0}=0$.

This is the (implicit) solution of the 2PI equations. We see that for real $\Psi$ the left hand side is real and positive, moreover for $\Psi(E=0)=1$. The $E<0$ part corresponds to imaginary values of $\Psi$. Since the equation itself is real, if $\Psi$ is a solution, it is $\Psi^{*}$, too. This means that the imaginary part is in fact the (half) discontinuity of the solution.

We see that irrespective of the value of $y_{0}$, at $E=0$, ie. on the mass shell $\Psi=1$ and so $\mathcal{G}=-\mathcal{G}_{0}$ finite. This yields difficulties when we try to apply renormalization conditions on the self-energy. Namely, if we keep the mass shell unchanged (this would correspond to the choice of $E_{0}$ above), then the renormalization of the self-energy would mean $\Sigma\left(p_{0}=m\right)=0$ and $\Sigma^{\prime}\left(p_{0}=m\right)=$ finite. Then, however, near the mass shell the propagator should always behave as $\sim 1 /\left(p_{0}-m\right)$, ie. infinite at the mass shell. This means that the physical renormalization process requires $\mathcal{G}_{0} \rightarrow \infty$. In this case the propagator behaves near the mass shell as:

$$
\mathcal{G}=\frac{-\mathcal{G}_{0}}{1+\mathcal{G}_{0} y_{0} \sqrt{\frac{2 \alpha}{\pi}}\left(m-p_{0}\right)} \stackrel{\substack{\mathcal{G}_{0} \rightarrow \infty \\ y_{0}=\sqrt{\pi /(2 \alpha)}}}{\longrightarrow} \frac{1}{p_{0}-m},
$$

because if $\Psi$ is close to 1 then the log term can be neglected in (38), and we find $\Psi=1+y_{0} E$.

For large values of $\Psi$, on the other hand, $y_{0}$ can be neglected. Then the integral can be evaluated as

$$
\sqrt{\pi} \operatorname{erfi}(\sqrt{\ln \Psi})=E
$$

For large $\Psi$ values it behaves as

$$
\frac{\Psi}{\sqrt{\ln \Psi}}=E, \quad \text { for large } E, \Psi
$$

\section{B. Numerical solution}

Now let us turn to the numerical study of the system, based on [6] and [22]: we determine the discontinuity of the self-energy self-consistently. The discontinuity of (30) now reads

$$
\underset{p_{0}}{\operatorname{Disc}} \Sigma(p)=\frac{\alpha}{\pi} \int_{0}^{p_{0}} d \omega\left(p_{0}-\omega\right) \varrho(\omega) .
$$

Knowing the discontinuity of the self-energy, we can use the Kramers-Kronig relation to restore the complete selfenergy:

$$
\Sigma(p)=\int_{-\infty}^{\infty} \frac{d \omega}{2 \pi} \frac{\operatorname{Disc}_{\omega} i \Sigma(\omega, \mathbf{k})}{p_{0}-\omega+i \varepsilon} .
$$

While (42) is a completely finite expression, in the Kramers-Kronig relation we will find divergences. This corresponds to the divergences of the self-energies which must be made finite by applying the appropriate counterterms. Technically one can regularize the integral in (43) and then make it finite with counterterms, or use the (twice) subtracted form of the Kramers Kronig relation. To see how it works, we determine the one-loop result from the tree level spectral function and the dimensional regularization of the Kramers-Kronig equations (interpreting $\omega \rightarrow \sqrt{\omega^{2}}$ ):

$$
-2 \alpha \mu^{2 \varepsilon} \int \frac{d^{1-2 \varepsilon} \omega}{(2 \pi)^{1-2 \varepsilon}} \omega\left(p_{0}-m-\omega\right)^{-1}=\frac{\alpha}{2 \pi}\left(p_{0}-m\right)\left[\frac{1}{\varepsilon}-2 \ln \frac{m-p_{0}}{\mu}+\ln \pi+1\right] .
$$


The divergence structure is the same, and also the $\overline{\mathrm{MS}}$ scheme result is the same as in (20) (the different finite parts are due to the different regularization method).

Now we can set up an algorithm to solve (42). We choose an arbitrary spectral function as a starting one (practically the free spectral function), then follow the following steps:

step 1:: compute the discontinuity of the self-energy using (42)

step 2:: compute the complete self-energy using the Kramers-Kronig relation (43)

step 3:: renormalize the self-energy with local counterterms. To fix the counterterms we used on-mass-shell (OM) renormalization scheme, ie. the real part of the self-energy at the mass shell is zero and its derivative is also zero

$$
\operatorname{Re} \Sigma\left(p_{0}=m\right)=0,\left.\quad \frac{d \operatorname{Re} \Sigma\left(p_{0}\right)}{d p_{0}}\right|_{p_{0}=m}=0 .
$$

We note here that releasing the first condition yields a mass shift, releasing the second condition yields a finite wave function renormalization. But in all renormalization schemes it will remain true that near the (renormalized) mass shell the propagator behaves as $\mathcal{G}\left(p_{0} \approx m\right)=\zeta /\left(p_{0}-m\right)$.

step 4:: construct the new spectral function from the discontinuity of the propagator knowing the real and imaginary part of the self-energy as

$$
\varrho(p)=\frac{2 \operatorname{Im} \Sigma(p)}{\left(p_{0}-m-\operatorname{Re} \Sigma(p)\right)^{2}+(\operatorname{Im} \Sigma(p))^{2}} .
$$

step 5:: continue with step 1 until the process converges.

Integrations in the above algorithm are performed numerically. This strategy was applied successfully for the $\Phi^{4}$ model in [6].

The direct application of this strategy, however, this times fails. Numerically what we can observe is that the spectral function becomes more and more shallow, and pointwise it goes to zero $\lim _{n} \varrho_{n}(p)=0$. In order to see a convergence, we had to use a supplementary step in the iteration after step 4:

step $4^{\prime}::$ use a rescaling of the generated spectral function:

$$
\varrho(p) \rightarrow A \varrho(B p)
$$

with appropriate $A$ and $B$ which can ensure convergence.

The appropriate values can be found by inspection, but the actual values are not too important (we used $A=73$ and $B=11$ in our numerics). In this way finally we succeeded to see convergence in the spectral function.

The numerical reason of this behavior is that the exact spectral function has a discontinuity at the mass shell, and - apart from this single point - it has always negative derivative. Numerically, however, we cannot have a jump, since in all regularizations equation (42) yields $\varrho\left(p_{0} \approx m\right) \sim\left(p_{0}-m\right)^{n}$ where $n \geq 2$. Since the exact curve starts to bend downwards, the recursion tries to lower the spectral function in order to have smaller derivative near the mass shell. Since the spectral function has to be positive, these requirements can be satisfied only with $\varrho=0$. With the continuous rescaling we can achieve that the numerically badly conditioned part, the vicinity of the mass shell, becomes smaller and smaller.

The numerical results can be seen on Figure1. The expected asymptotics can be nicely identified on the calculation (cf. Fig. 2). This also proves implicitly that the strategy to resolve the aforementioned numerical problem with the 2PI equation was correct.

If we compare the 1-loop, the $2 \mathrm{PI}$ and the exact results we see that there is not too much improvement. The IR problem near the mass shell which made the 1-loop calculation unreliable, seems to be cured, but in fact the result is not closer to the exact one as the one-loop result. The physics of the deep infrared photons cannot be described by the $2 \mathrm{PI}$ approximation. 

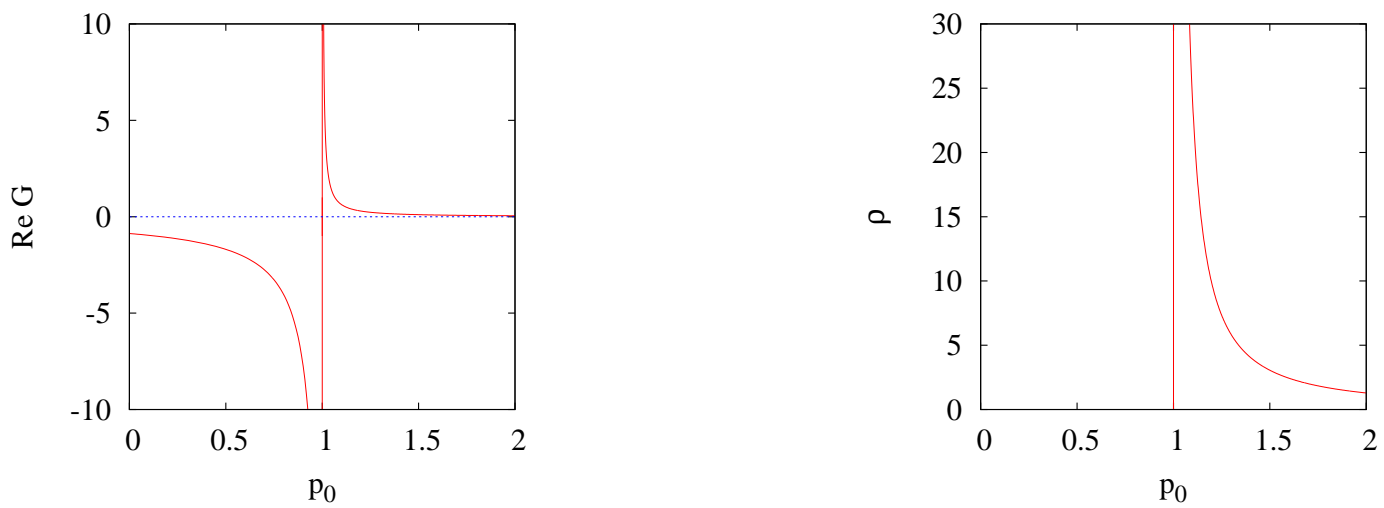

FIG. 1. The real part and discontinuity of the $2 \mathrm{PI}$ propagator.

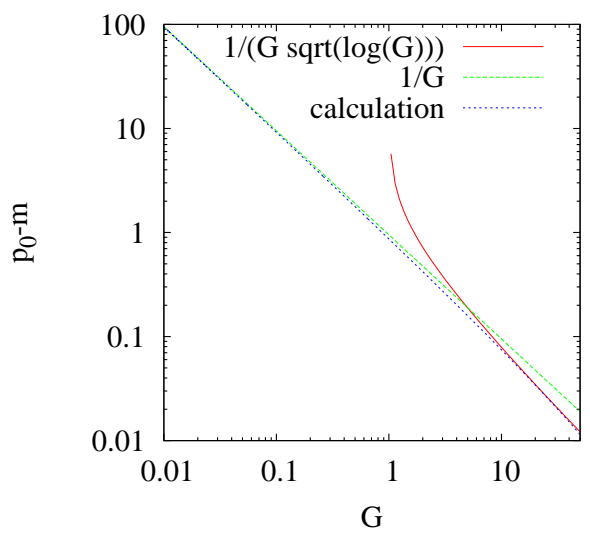

FIG. 2. The expected asymptotics plotted on the data

\section{SCHWINGER-DYSON EQUATIONS AND WARD-IDENTITIES}

The next level of the approximations is based on the Schwinger-Dyson equations. For the Bloch-Nordsieck model in Feynman gauge it can be written as

$$
\Sigma(p)=-i e^{2} \int \frac{d^{4} k}{(2 \pi)^{4}} G(k) \mathcal{G}(p-k) u_{\mu} \Gamma^{\mu}(k ; p-k, p),
$$

where $\Gamma^{\mu}$ is the vertex function.

For the vertex function there is another exact equation, coming from the current conservation. This results in the Ward-identity analogous to the QED case [24]:

$$
k_{\mu} \Gamma^{\mu}(k ; p-k, p)=\mathcal{G}^{-1}(p)-\mathcal{G}^{-1}(p-k) .
$$

In this model, however, the vertex function is proportional to $u^{\mu}$. In principle the Lorentz-index in this model can come from $u^{\mu}$ or from any of the momenta. But, since the fermion propagator depends on the 4-momentum in the form $u_{\mu} p^{\mu}$, the fermion-photon vertex does not depend on the momentum components which are orthogonal to $u_{\mu}$. Therefore the Lorentz-index which comes from $q^{\mu}$ in fact comes from the longitudinal part of $q^{\mu}$, ie. proportional to $u^{\mu}$. So we can write $\Gamma^{\mu}(k ; p, q)=u^{\mu} \Gamma(k ; p, q)$.

This gives us the possibility that from the Ward identities we exactly determine the vertex function. The Wardidentity for the current conservation yields then in case when $u=(1,0,0,0)$ :

$$
k_{\mu} \Gamma^{\mu}(k ; p-k, p)=k_{0} \Gamma(k ; p-k, p)=\mathcal{G}^{-1}(p)-\mathcal{G}^{-1}(p-k) \quad \Rightarrow \quad \Gamma(k ; p-k, p)=\frac{\mathcal{G}^{-1}(p)-\mathcal{G}^{-1}(p-k)}{k_{0}} .
$$


Therefore we find

$$
\Sigma(p)=-i e^{2} \int \frac{d^{4} k}{(2 \pi)^{4}} \frac{G(k)}{k_{0}} \mathcal{G}(p-k)\left(\mathcal{G}^{-1}(p)-\mathcal{G}^{-1}(p-k)\right) .
$$

This is an exact equation in the Bloch-Nordsieck model. Now we will solve this equation in the renormalized theory, and demonstrate that the solution is indeed identical with the Bolch-Nordsieck solution presented in Section [II]

In the second term $\mathcal{G}^{-1}(p-k)$ drops out, resulting in an integral

$$
-i e^{2} \int \frac{d^{4} k}{(2 \pi)^{4}} \frac{G(k)}{k_{0}}=0
$$

because of $k_{0} \rightarrow-k_{0}$ symmetry. What remains is

$$
\Sigma(p)=\mathcal{G}^{-1}(p)\left(-i e^{2}\right) \int \frac{d^{4} k}{(2 \pi)^{4}} \frac{G(k)}{k_{0}} \mathcal{G}(p-k) .
$$

This form is true in the original model, we shall now find the renormalized form. First we adapt the wave function renormalization for the fermionic fields which changes the bare propagator to $1 /\left(Z p_{0}-(m+\delta m)\right)$ where $Z=1+\delta Z$. We will assume that the mass shell remains the same, then $m+\delta m=Z m$, and the free propagator will be $1 /\left(Z\left(p_{0}-m\right)\right)$. We will use also the notation $e_{b}=e+\delta e$. The full propagator then reads

$$
\mathcal{G}^{-1}(p)=Z\left(p_{0}-m\right)-\Sigma(p),
$$

Using (53) we find the equation

$$
\mathcal{G}(p)=\frac{\zeta\left(p_{0}\right)}{p_{0}-m+i \varepsilon}
$$

where

$$
\zeta\left(p_{0}\right)=\frac{1+J\left(p_{0}\right)}{Z} \quad \text { and } \quad J\left(p_{0}\right)=-i e_{b}^{2} \int \frac{d^{4} k}{(2 \pi)^{4}} \frac{G(k)}{k_{0}} \mathcal{G}(p-k) .
$$

$\zeta\left(p_{0}\right)$ can be interpreted as a running wave function renormalization constant.

With a spectral representation

$$
J\left(p_{0}\right)=\int_{0}^{\infty} \frac{d \omega}{2 \pi} \varrho(\omega) I_{1}\left(\omega-p_{0}+i \varepsilon\right), \quad \text { where } \quad I_{1}(a)=i e^{2} \int \frac{d^{4} k}{(2 \pi)^{4}} \frac{1}{k_{0}^{2}-\mathbf{k}^{2}+i \varepsilon} \frac{1}{k_{0}} \frac{1}{a+k_{0}} .
$$

In the Appendix we evaluate $I_{1}\left(p_{0}\right)$, and we find

$$
J\left(p_{0}\right)=\frac{e_{b}^{2}}{4 \pi^{2}} \int_{-\infty}^{\infty} \frac{d \omega}{2 \pi} \varrho(\omega)\left[\mathcal{D}_{\varepsilon}-\ln \frac{\omega-p_{0}+i \varepsilon}{\mu}\right] .
$$

We rewrite it into (56), then, assuming normalizable spectral function, after some algebraic manipulation we find

$$
=\frac{\frac{1}{\alpha_{b}}+\frac{1}{\pi} \mathcal{D}_{\varepsilon}-\frac{1}{\pi} \int_{-\infty}^{\infty} \frac{d \omega}{2 \pi} \varrho(\omega) \ln \frac{\omega-p_{0}+i \varepsilon}{\mu}}{Z / \alpha_{b}}
$$

We may assume that the explicit integral is not UV divergent (it can be checked a posteriori, or, as in the present case, knowing the exact solution). Then the above equation can be made finite by requiring

$$
\frac{1}{\alpha_{b}}+\frac{1}{\pi} \mathcal{D}_{\varepsilon}=\frac{1}{\alpha_{r}}, \quad \frac{Z}{\alpha_{b}}=\frac{z_{r}}{\alpha_{r}}
$$

where $\alpha_{r}$ and $z_{r}$ are finite. This form can be interpreted physically as the appearance of the renormalized coupling $\alpha_{r}$ and the finite wave function renormalization $z_{r}$. We note that the coupling constant renormalization equation agrees with the nonperturbative coupling constant renormalization in the $\mathrm{O}(\mathrm{N})$ models [23]. 
Now we find

$$
\zeta\left(p_{0}\right)=\frac{1}{z_{r}}\left(1-\frac{\alpha_{r}}{\pi} \int_{-\infty}^{\infty} \frac{d \omega}{2 \pi} \varrho(\omega) \ln \frac{\omega-p_{0}-i \varepsilon}{\mu}\right) .
$$

This function depends on the arbitrary scale $\mu$, but the physics, of course, must be $\mu$ independent. This can be achieved by appropriately changing the $z_{r}$ and $\alpha_{r}$ constant when we change $\mu$. The $\mu$-independence of $\zeta\left(p_{0}\right)$ requires (using the sum rule for $\varrho$ ):

$$
\frac{d \zeta\left(p_{0}\right)}{d \ln \mu}=-\frac{1}{z_{r}^{2}} \frac{d z_{r}}{d \ln \mu}\left(1-\frac{\alpha_{r}}{\pi} \int_{-\infty}^{\infty} \frac{d \omega}{2 \pi} \varrho(\omega) \ln \frac{\omega-p_{0}-i \varepsilon}{\mu}\right)-\frac{1}{z_{r} \pi} \frac{d \alpha_{r}}{d \ln \mu} \int_{-\infty}^{\infty} \frac{d \omega}{2 \pi} \varrho(\omega) \ln \frac{\omega-p_{0}-i \varepsilon}{\mu}+\frac{1}{z_{r}} \frac{\alpha_{r}}{\pi}=0 .
$$

This can be satisfied if

$$
-\frac{1}{z_{r}^{2}} \frac{d z_{r}}{d \ln \mu}+\frac{1}{z_{r}} \frac{\alpha_{r}}{\pi}=0, \quad \frac{1}{z_{r}^{2}} \frac{d z_{r}}{d \ln \mu} \frac{\alpha_{r}}{\pi}-\frac{1}{z_{r} \pi} \frac{d \alpha_{r}}{d \ln \mu}=0 .
$$

The second equation means $z_{r}=\alpha_{r} / \alpha_{0}$ where $\alpha_{0}$ is a constant; the first equation then reads

$$
\frac{d \ln z_{r}}{d \ln \mu}=\frac{\alpha_{r}}{\pi} \Rightarrow \frac{d \alpha_{r}}{d \ln \mu}=\frac{\alpha_{r}^{2}}{\pi} \Rightarrow-\frac{1}{\alpha_{r}(\mu)}+\frac{1}{\alpha_{r}\left(\mu_{0}\right)}=\frac{1}{\pi} \ln \frac{\mu}{\mu_{0}} \Rightarrow \alpha_{r}(\mu)=\frac{\alpha_{r}\left(\mu_{0}\right)}{1+\frac{\alpha_{r}\left(\mu_{0}\right)}{\pi} \ln \frac{\mu_{0}}{\mu}}
$$

Using the normalizability of $\varrho$ we finally find

$$
\zeta\left(p_{0}\right)=\frac{\alpha_{0}}{\pi} \int_{-\infty}^{\infty} \frac{d \omega}{2 \pi} \varrho(\omega) \ln \frac{\Lambda}{\omega-p_{0}-i \varepsilon}, \quad \Lambda=\mu e^{\frac{\pi}{\alpha_{r}}} .
$$

The $\alpha_{0}$ and the scale $\Lambda$ are renormalization group independent quantities (ie. independent of the scale $\mu$ ), these characterize the renormalization scheme. The appearance of a scale $\Lambda$ is the manifestation of dimensional transmutation. Now, instead of that scale $\Lambda$ it is worth to use $M$ for which $\operatorname{Re} \zeta(M)=0$. Clearly $M \approx \Lambda$ if $\Lambda \gg m$. Then with differentiating $\zeta$ with respect to $p_{0}$ we find

$$
\frac{d \zeta\left(p_{0}\right)}{d p_{0}}=-\frac{\alpha_{0}}{\pi} \int_{-\infty}^{\infty} \frac{d \omega}{2 \pi} \frac{\varrho(\omega)}{p_{0}-\omega+i \varepsilon}=-\mathcal{G}\left(p_{0}\right) \Rightarrow \zeta\left(p_{0}\right)=\frac{\alpha_{0}}{\pi} \int_{p_{0}}^{M} d \omega \mathcal{G}(\omega) .
$$

This gives finally

$$
\left(p_{0}-m\right) \mathcal{G}(p)=\frac{\alpha_{0}}{\pi} \int_{p_{0}}^{M} d \omega \mathcal{G}(\omega) .
$$

By differentiation with respect to $p_{0}$ we find

$$
\left(p_{0}-m\right) \mathcal{G}^{\prime}+\mathcal{G}=-\frac{\alpha_{0}}{\pi} \mathcal{G} \Rightarrow \mathcal{G}(p)=g_{0}\left(p_{0}-m\right)^{-1-\frac{\alpha_{0}}{\pi}},
$$

where $g_{0}$ is an arbitrary constant. This is indeed the solution of Bloch and Nordsieck (14), now in terms of the renormalized quantities.

But we also see that the condition $\mathcal{G}\left(p_{0}=M\right)=0$ can be satisfied only with $g_{0}=0$. This is in close relation with the fact that at the mass shell $p_{0} \approx m$, the propagator (and its discontinuity) is not integrable.

The lesson of this analysis is that the deep IR physics is well describable by the Schwinger-Dyson equation, truncated in a way which respects the Ward-identities. As we have seen, this strategy is renormalizable and exact in case of the Bloch-Nordsieck model.

A big advantage of this approach is that, besides being exact in the IR, it can be easily generalized to other theories. So we expect that in QED the Schwinger-Dyson equations truncated in the way we have done it in the Bloch-Nordsieck theory will represent the exact result well in the problematic deep IR regime. 


\section{CONCLUSIONS}

In this paper we examined the exactly solvable Bloch-Nordsieck model from the point of view of different perturbative methods. We first reviewed the known method to obtain the exact solution [17]. Then the different levels of approximations, like the one-loop level perturbation theory, the 2PI resummation and the truncated Schwinger-Dyson equations were studied. The 1-loop result exhibits an IR sensitivity when we approach the mass shell which renders the theory ill-defined. The self-energy (2PI) resummation reorganizes the perturbative series in a way that this IR problem disappears. This does not mean, however, that the result itself would be closer to the exact one, only the explicit IR sensitivity cannot be seen. On the other hand, the Schwinger-Dyson equations, truncated in a way that the Ward-identities are satisfied yield the exact result in the Bloch-Nordsieck model. This is a new way of obtaining the exact solution in the Bloch-Nordsieck model. And, while the original solution method is very hard to generalize to other theories, the generalization of the specially truncated Schwinger-Dyson equations is straightforward.

\section{ACKNOWLEDGMENTS}

The authors thank useful discussions with T.S. Bíró, F. Csikor and A. Patkós. This work is supported by the Hungarian Research Fund (OTKA) under contract No. K68108.

\section{Appendix A: Details of the one loop calculation}

The one-loop contribution to the self-energy reads, with a generic $u$ vector in Feynman gauge:

$$
\Sigma=-i e^{2} u^{2} \int \frac{d^{4} k}{(2 \pi)^{4}} \frac{1}{k^{2}+i \varepsilon} \frac{1}{u^{\mu}\left(p_{\mu}-k_{\mu}\right)-m+i \varepsilon} .
$$

This is Lorentz-invariant, if we a Lorentz transformation both on $u$ and $p$. So we may choose a special frame where $\Lambda u=\left(u_{0}, 0,0,0\right)$. If $u$ is a proper 4 -velocity, then $u_{0}=1$; if it is $u=(1, \mathbf{v})$, then $u_{0}=\sqrt{1-\mathbf{v}^{2}}$, but still constant, since $\nu$ is a parameter of the theory. We find then

$$
\Sigma=e^{2} u_{0} I_{0}\left(\frac{m}{u_{0}}-p_{0}-i \varepsilon\right), \quad I_{0}(a)=i \int \frac{d^{4} k}{(2 \pi)^{4}} \frac{1}{k^{2}+i \varepsilon} \frac{1}{a+k_{0}} .
$$

Thus it is enough to consider $I_{0}$ only. There we transform to positive frequency integrals

$$
I_{0}(a)=i \int \frac{d^{4} k}{(2 \pi)^{4}} \frac{1}{k^{2}+i \varepsilon} \frac{1}{a+k_{0}}=\frac{i a}{\pi} \int_{0}^{\infty} d k_{0} \int \frac{d^{3} \mathbf{k}}{(2 \pi)^{3}} \frac{1}{k_{0}^{2}-\mathbf{k}^{2}+i \varepsilon} \frac{1}{a^{2}-k_{0}^{2}}=\frac{a}{\pi} \int_{0}^{\infty} d k_{0} \int \frac{d^{3} \mathbf{k}}{(2 \pi)^{3}} \frac{1}{k_{0}^{2}+\mathbf{k}^{2}} \frac{1}{a^{2}+k_{0}^{2}},
$$

where in the last step we performed Wick rotation (the choice of the imaginary part of $a$ is crucial for the direction of the rotation on the complex plane).

Now we can write up the integral in $k_{0}$ and $\mathbf{k}$ space, in the latter using $3-2 \varepsilon$ dimensions:

$$
I_{0}=a \mu^{2 \varepsilon} \int_{0}^{\infty} \frac{d k_{0}}{\pi} \int \frac{d^{3-2 \varepsilon} \mathbf{k}}{(2 \pi)^{3-2 \varepsilon}} \frac{1}{k_{0}^{2}+\mathbf{k}^{2}} \frac{1}{a^{2}+k_{0}^{2}}
$$

We use the relation

$$
\mu^{2 \varepsilon} \int \frac{d^{d-2 \varepsilon} k}{(2 \pi)^{d-2 \varepsilon}} f\left(k^{2}\right)=\frac{2\left(4 \pi \mu^{2}\right)^{\varepsilon}}{(4 \pi)^{d / 2} \Gamma(d / 2-\varepsilon)} \int_{0}^{\infty} d k k^{d-1+2 \varepsilon} f\left(k^{2}\right)=\frac{\left(4 \pi \mu^{2}\right)^{\varepsilon}}{(4 \pi)^{d / 2} \Gamma(d / 2-\varepsilon)} \int_{0}^{\infty} d z z^{\frac{d}{2}-1-\varepsilon} f(z)
$$

to proceed as

$$
\begin{aligned}
I_{0} & =\frac{a}{\pi} \int_{0}^{\infty} d k_{0} \frac{1}{a^{2}+k_{0}^{2}} \frac{\left(4 \pi \mu^{2}\right)^{\varepsilon}}{(4 \pi)^{3 / 2} \Gamma\left(\frac{3}{2}-\varepsilon\right)} \int_{0}^{\infty} d z z^{\frac{3}{2}-1-\varepsilon}\left(k_{0}^{2}+z\right)^{-1}=\frac{a\left(4 \pi \mu^{2}\right)^{\varepsilon} \Gamma\left(-\frac{1}{2}+\varepsilon\right)}{8 \pi^{2} \sqrt{\pi}} \int_{0}^{\infty} d k_{0} \frac{k_{0}^{1-2 \varepsilon}}{a^{2}+k_{0}^{2}}= \\
& =\frac{a \Gamma\left(-\frac{1}{2}+\varepsilon\right) \Gamma(1-\varepsilon)}{16 \pi^{2} \sqrt{\pi}}\left(\frac{4 \pi \mu^{2}}{a^{2}}\right)^{\varepsilon} \Gamma(\varepsilon)=\frac{-a}{8 \pi^{2}}\left[\frac{1}{\varepsilon}-2 \ln \frac{a}{\mu}+2+\ln \pi-\gamma_{E}\right] .
\end{aligned}
$$


We write it as

$$
I_{0}=\frac{-a}{4 \pi^{2}}\left[\mathcal{D}_{\varepsilon}-\ln \frac{a}{\mu}\right]
$$

where

$$
\mathcal{D}_{\varepsilon}=\frac{1}{2 \varepsilon}+1+\frac{\ln \pi-\gamma_{E}}{2} .
$$

Therefore

$$
\Sigma=\left(u_{0} p_{0}-m\right) \frac{e^{2}}{8 \pi^{2}}\left[\frac{1}{\varepsilon}-2 \ln \frac{u_{0} p_{0}-m}{u_{0} \mu}+2+\ln \pi-\gamma_{E}\right]
$$

We also need to compute

$$
I_{1}(a)=i \int \frac{d^{4} k}{(2 \pi)^{4}} \frac{1}{k^{2}+i \varepsilon} \frac{1}{k_{0}} \frac{1}{a+k_{0}}=\frac{-i}{\pi} \int_{0}^{\infty} d k_{0} \int \frac{d^{3} \mathbf{k}}{(2 \pi)^{3}} \frac{1}{k_{0}^{2}-\mathbf{k}^{2}+i \varepsilon} \frac{1}{a^{2}-k_{0}^{2}}=-\frac{1}{a} I_{0}(a)=\frac{1}{4 \pi^{2}}\left[\mathcal{D}_{\varepsilon}-\ln \frac{a}{\mu}\right] \text {. }
$$

[1] J. M. Luttinger and J. C. Ward, Phys. Rev. 118 (1960) 1417. G. Baym, Phys. Rev. 127 (1962) 1391. J. M. Cornwall, R. Jackiw and E. Tomboulis, Phys. Rev. D 10 (1974) 2428.

[2] H. van Hees an J. Knoll, Phys. Rev. D65 (2002) 025010 hep-ph/0107200; Phys. Rev. D65 (2002) 105005 hep-ph/0111193; Phys. Rev. D66 (2002) 025028 hep-ph/0203008

[3] J.-P. Blaizot, E. Iancu and U. Reinosa, Phys. Lett. B568 (2003) 160 hep-ph/0301201]; Nucl. Phys. A736 (2004) 149 hep-ph/0312085

[4] J. Berges, Sz. Borsanyi, U. Reinosa and J. Serreau, Annals Phys. 320 (2005) 344 hep-ph/0503240

[5] U. Reinosa, J. Serreau, JHEP 0607 (2006) 028 hep-th/0605023;

[6] A. Jakovac, Phys. Rev. D76, 125004 (2007). hep-ph/0612268.

[7] A. Patkos, Z. .Szep, Nucl. Phys. A811, 329-352 (2008). arXiv:0806.2554 [hep-ph]].

[8] J. Berges and J. Cox, Phys. Lett. B 517 (2001) 369.

[9] J. Berges, S. Borsanyi and C. Wetterich, Phys. Rev. Lett. 93 (2004) 142002.

[10] J. Berges, S. .Borsanyi, U. Reinosa, J. Serreau, Phys. Rev. D71, 105004 (2005). hep-ph/0409123.

[11] A. Jakovac, Phys.Rev. D78 (2008) 085013, arXiv:0808.1800 [hep-th]]

[12] G. Fejos, A. Patkos, Z. .Szep, Phys. Rev. D80, 025015 (2009). arXiv:0902.0473 [hep-ph]].

[13] U. Reinosa, J. Serreau, Annals Phys.325:969-1017,2010 arXiv:0906.2881

[14] Sz. Borsanyi, U. Reinosa, Phys.Lett.B661:88-94,2008, arXiv:0709.2316

[15] J. Berges, S. Roth, Nucl.Phys.B847:197-219,2011, arXiv:1012.1212.

[16] M.E. Carrington, E. Kovalchuk, Phys.Rev.D76:045019,2007, arXiv:0705.0162

[17] N.N. Bogoliubov and D.V. Shirkov, Introduction to the theories to the quantized fields (John Wiley \& Sons, Inc., 1980)

[18] H.M. Fried, Greens Functions and Ordered Exponentials (Cambridge University Press, 2002)

[19] J. -P. Blaizot and E. Iancu, Phys. Rev. D 56, 7877 (1997) hep-ph/9706397, J. -P. Blaizot and E. Iancu, Phys. Rev. D 55, 973 (1997) hep-ph/9607303.

[20] H.A. Weldon, Phys. Rev. D44 (1991) 3955; Phys. Rev. D49 (1994) 1579.

[21] M. Le Bellac, Thermal Field Theory, (Cambridge Univ. Press, 1996.)

[22] P. Mati diplomawork

[23] J.O. Andersen, D. Boer, H.J. Warringa, Phys. Rev. D70 (2004), 116007 hep-ph/0408033, A. Jakovac, Phys. Rev. D 78, 085013 (2008) arXiv:0808.1800 [hep-th]], G. Fejos, A. Patkos and Z. .Szep, Phys. Rev. D 80, 025015 (2009) arXiv:0902.0473 [hep-ph]].

[24] M.E. Peskin, D.V. Schroeder, An Introduction to Quantum Field Theory, (Perseus Books Publishing, 1995.) 